TITLE:

\title{
Effects of exposure to hyperbaric oxygen on oxidative stress in rats with type II collagen-induced arthritis.
}

\section{AUTHOR(S):}

Nagatomo, F; Gu, N; Fujino, H; Okiura, T;

Morimatsu, F; Takeda, I; Ishihara, Akihiko

\section{CITATION:}

Nagatomo, F ...[et al]. Effects of exposure to hyperbaric oxygen on oxidative stress in rats with type II collagen-induced arthritis.. Clinical and experimental medicine 2010, 10(1): 713

\section{ISSUE DATE:}

2010-03

URL:

http://hdl.handle.net/2433/97894

\section{RIGHT:}

c 2009 Springer-Verlag.; This is not the published version. Please cite only the published version.; この論文は出版社版でありません。引用の 際には出版社版をご確認ご利用ください。 
Clinical and Experimental Medicine (Original Article)

\section{Effects of exposure to hyperbaric oxygen on oxidative stress in rats with type II collagen-induced arthritis}

Nagatomo $\mathrm{F}^{1}$, Gu $\mathrm{N}^{1}$, Fujino $\mathrm{H}^{2}$, Okiura $\mathrm{T}^{3}$, Morimatsu $\mathrm{F}^{3}$, Takeda $\mathrm{I}^{4}$, Ishihara $\mathrm{A}^{1}$

${ }^{1}$ Laboratory of Neurochemistry, Graduate School of Human and Environmental

Studies, Kyoto University, Kyoto 606-8501, Japan

${ }^{2}$ Division of Rehabilitation Sciences, Kobe University Graduate School of Health

Sciences, Kobe 654-0142, Japan

${ }^{3}$ Research and Development Center Nippon Meat Packers Inc., Tsukuba 300-2646, Japan

${ }^{4}$ Department of Physical Therapy Faculty of Health Care Science, Himeji Dokkyo University, Himeji 670-8524, Japan

Correspondence: Prof. Akihiko Ishihara, Laboratory of Neurochemistry,

Graduate School of Human and Environmental Studies, Kyoto University,

Sakyo-ku, Kyoto 606-8501, Japan

E-mail: ishihara@life.mbox.media.kyoto-u.ac.jp

Phone:: +81-75-753-6881; FAX: +81-75-753-6771 
Abstract Arthritis was induced in 9-week-old female Dark Agouti rats by injecting type II collagen. Serum levels of the derivatives of reactive oxygen metabolites (dROMs), which are oxidative stress markers, and C-reactive protein $(\mathrm{CRP})$ in arthritic rats that were exposed to a pressure of 1.25 atmospheres absolute and an oxygen concentration of $36 \%$ for 3 weeks (arthritis + HBO group) were compared to those of control rats (control group) and arthritic rats that were not exposed to hyperbaric oxygen (arthritis group). The body weights of the arthritis and arthritis + HBO groups were lower than that of the control group, whereas no difference in the body weight was observed between the arthritis and arthritis + HBO groups. The serum levels of dROMs and CRP in the arthritis group were higher than those in the control and arthritis $+\mathrm{HBO}$ groups. No difference in the serum level of CRP was observed between the control and arthritis $+\mathrm{HBO}$ groups. These results indicate that the conditions of hyperbaric oxygen exposure used in this study are effective for reducing the levels of reactive oxygen species, which are overproduced during arthritis.

Key words: C-reactive protein; derivatives of reactive oxygen metabolites; hyperbaric oxygen exposure; rat; reactive oxygen species; type II collagen-induced arthritis 


\section{Introduction}

Highly reactive and toxic transient chemical species, referred to as reactive oxygen species (ROS), are overproduced by phagocytes during the development and progression of inflammatory processes and pathogenesis in a number of diseases [1]. The excessive production of ROS can damage cellular components such as nucleic acids, lipids, proteins, membranes, and matrix components. In addition, ROS serve as important intracellular signaling and regulatory molecules that enhance the synovial inflammatory-proliferative response [2]. Oxygen metabolism plays an important role in the pathogenesis of many joint diseases. The potential sources of ROS production are numerous in the case of joint diseases. In degenerative joint diseases, proinflammatory factors such as cytokines and prostaglandins are released at sites of inflammation and destruction together with ROS [3]. The production of tumor necrosis factor (TNF)- $\alpha$ contributes to excessive ROS release and causes substantial reduction in the activity of antioxidant enzymes in inflammatory joints. Several other proinflammatory cytokines, including interleukin (IL)-1, IL-6, and IL-8, are produced by synovial cells; these cytokines recruit neutrophils into the synovial fluids and exert their effects by activating the intracellular signaling pathways [4]. C-reactive protein (CRP) is a marker of inflammation and destruction of tissues such as cartilage and bone. The serum level of CRP correlates with the production of derivatives of reactive oxygen metabolites (dROMs), which are oxidative stress markers $[5,6]$.

An elevation in atmospheric pressure accompanied by an increase in oxygen concentration enhances the partial pressure of oxygen and increases the concentration of dissolved oxygen in the plasma. We determined that a 
pressure of 1.25 atmospheres absolute (ATA) and an oxygen concentration of $36 \%$ are required for obtaining effective responses with regard to oxidative metabolism [7, 8]. Previous studies $[9,10]$ have reported low levels of partial pressure of oxygen in the synovial fluid of arthritic joints. These joints are characterized by hypoxia, which is caused by increased oxygen demand and decreased blood flow [11-13]. Hypoxic conditions induce the production of proinflammatory cytokines and excessive ROS release in inflammatory joints. We hypothesized that increased pressure and enhanced delivery and uptake of oxygen induced by exposure to hyperbaric oxygen are effective in reducing arthritis-induced inflammation because exposure to hyperbaric oxygen may prevent and improve hypoxic conditions in inflammatory joints.

In this study, we compared the serum levels of dROMs, TNF- $\alpha$, IL-6, and CRP in arthritic rats that were exposed to hyperbaric oxygen to those of control rats and arthritic rats that were not exposed to hyperbaric oxygen. We verified that the conditions of hyperbaric oxygen exposure used in this study are effective for decreasing the levels of ROS, which are overproduced during arthritis.

\section{Materials and methods}

All experimental procedures and animal care were conducted in accordance with the guidelines stated in the Guide for the Care and Use of Laboratory Animals issued by the Institutional Animal Experimentation Committee of Kyoto University.

\section{Experimental animals}


Bovine type II collagen (CII) was dissolved in $0.1 \mathrm{M}$ acetic acid at a concentration of $2 \mathrm{mg} / \mathrm{ml}$ by stirring overnight at $4^{\circ} \mathrm{C}$. Dissolved $\mathrm{Cll}$ was frozen at a temperature of $-70^{\circ} \mathrm{C}$ until use. We immunized 9-week-old female Dark Agouti (DA) rats $(n=12)$, which are susceptible to induction of adjuvant arthritis, with an emulsion of $2 \mathrm{mg} / \mathrm{ml}$ of $\mathrm{CII}$ in incomplete Freund's adjuvant (IFA). The emulsions were prepared by homogenizing 1 part of dissolved Cll into 1 part of IFA placed in an ice-water bath. The rats were anesthetized by an intraperitoneal injection of sodium pentobarbital (50 mg/kg body weight); thereafter, $0.2 \mathrm{ml}$ of the emulsion was intradermally injected into 3-5 other sites located on the back of each rat. Following injection, half of these rats (arthritis + HBO group; $n=6)$ were exposed to a pressure of 1.25 ATA $(950 \mathrm{mmHg})$ and an oxygen concentration of $36 \%$, automatically maintained by a computer-assisted system, for 3 weeks, while the other rats (arthritis group; $n=$ 6) were placed in a hyperbaric chamber under normal conditions (1 ATA (760 $\mathrm{mmHg}$ ) and $21 \%$ oxygen concentration); therefore, this group was not exposed to hyperbaric oxygen. Nonimmunized DA rats (control group; $n=6$ ) served as controls. All rats were individually housed in same-sized cages in a room maintained under controlled 12-h light-dark cycles (lights switched off from 2000 to 0800 ) at a temperature of $22 \pm 2{ }^{\circ} \mathrm{C}$ with a relative humidity of $45-65 \%$. Food and water were provided ad libitum to all groups. The body weight of each rat was measured every week. The pictures of the paws in all rats were taken when they were 12 weeks old. The arthritis severity was determined by means of visual examination, on the basis of the number of instances of knuckle swelling (0 point, no sign; 1 point, 1 or 2 digits; 2 points, 3 digits; 3 points, 4 digits; and 4 points, all digits), and the degree of arch edema (0 point, no sign; 1 
point, light; 2 points, mild; 3 points, heavy; and 4 points, severe). The arthritis score of each rat was calculated by determining the average of the points assigned to individual paws. Therefore, the lowest arthritis score corresponded to 0 points, while the highest arthritis score corresponded to 8 points.

\section{Blood sampling and biochemical measurements}

Following $12 \mathrm{~h}$ of fasting, blood samples were collected from the abdominal aorta of the rats anesthetized by an intraperitoneal injection of sodium pentobarbital (50 mg/kg body weight). The blood samples were centrifuged $(6,000 \mathrm{~g} ; 1 \mathrm{~min})$ and evaluated photometrically. We used a device capable of determining the levels of free radicals and antioxidant potentials (Free Radical Analytical System 4; Health \& Diagnostics; Grosseto, Italy) to measure the serum level of dROMs [14]. The serum level of dROMs was used as an index to determine the level of oxidative stress induced by measuring the amount of organic hydroperoxide $(\mathrm{ROOH})$ converted into radicals that oxidize N,N-diethyl-para-phenylenediamine. The serum levels of TNF- $\alpha$ (R\&D Systems, Inc.; MN, USA), IL-6 (R\&D Systems, Inc.), and CRP (Helica Biosystems, Inc.; CA, USA) were measured by a routine laboratory method using the enzyme-linked immunosorbent assay (ELISA) kit.

\section{Statistical analysis}

Values are presented as mean and standard deviation. One-way analysis of variance (ANOVA) was used to evaluate the differences among groups. When the differences were found to be significant, further comparisons were made by performing post hoc tests. A probability level of 0.05 was 
considered to be statistically significant.

\section{Results}

\section{Body weight}

No difference in the body weight was observed among the control, arthritis, and arthritis + HBO groups at 9, 10, and 11 weeks of age (Figure 1). In contrast, the body weights of the 12-week-old arthritis and arthritis $+\mathrm{HBO}$ groups were lower than that of the age-matched control group.

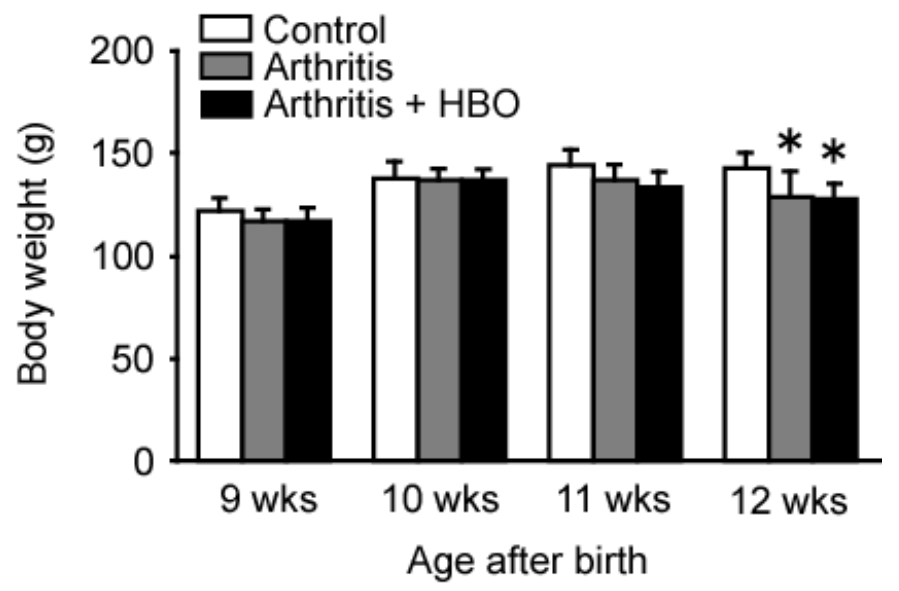

Fig. 1 The body weights of control rats and arthritic rats that were and were not exposed to hyperbaric oxygen. Data are presented as mean and standard deviation as determined from 6 animals. $\mathrm{HBO}$, hyperbaric oxygen exposure. $\quad{ }^{*} P<0.05$ compared to age-matched control rats.

\section{Arthritis severity}

The rats in the control group were not arthritic (Fig. 2, A1-6). In contrast, the rats in the arthritis (Fig. 2, B1-6) and arthritis + HBO (Fig. 2, C1-6) groups presented with knuckle swelling and/or arch edema. No difference in 
the arthritis score was observed between the arthritis $(6.0 \pm 2.3, n=6)$ and arthritis + HBO $(4.8 \pm 1.6, n=6)$ groups.
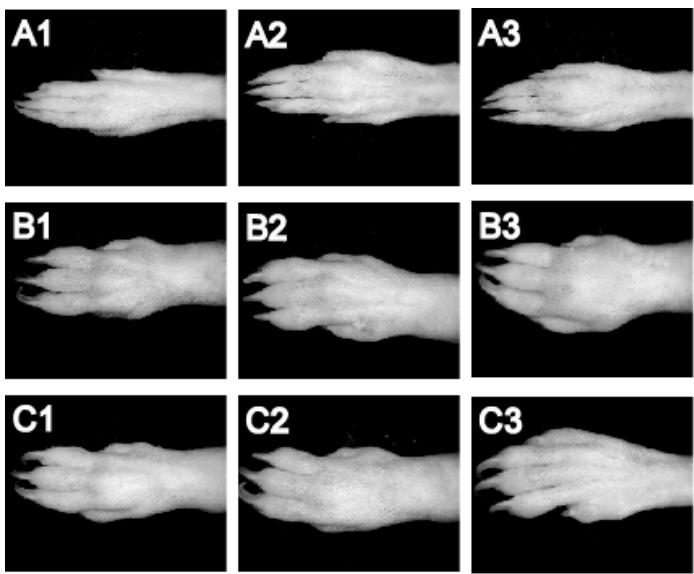

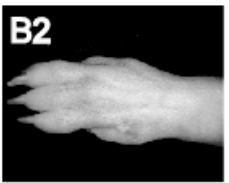

C2

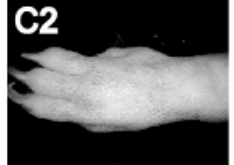

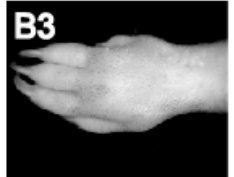

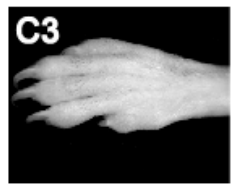

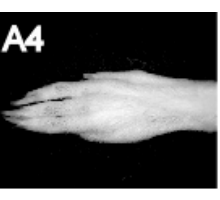
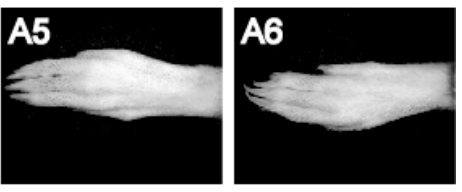
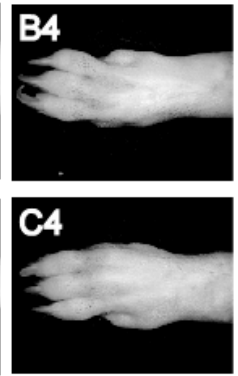

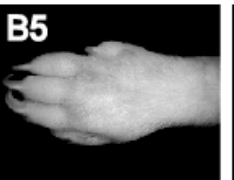

C5

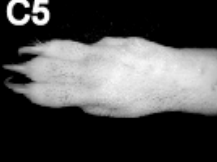

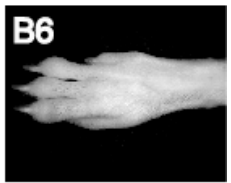

C6

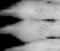

Fig. 2 The hind-paws of control $(A 1-6)$ and arthritic rats that were $(C 1-6)$ and were not (B1-6) exposed to hyperbaric oxygen. The scale bar on C6 indicates $1 \mathrm{~cm}$.

\section{Serum level of dROMs}

The serum levels of dROMs in the arthritis and arthritis + HBO groups were higher than that in the control group (Figure $3 \mathrm{~A}$ ). The serum level of dROMs in the arthritis + HBO group was lower than that in the arthritis group.
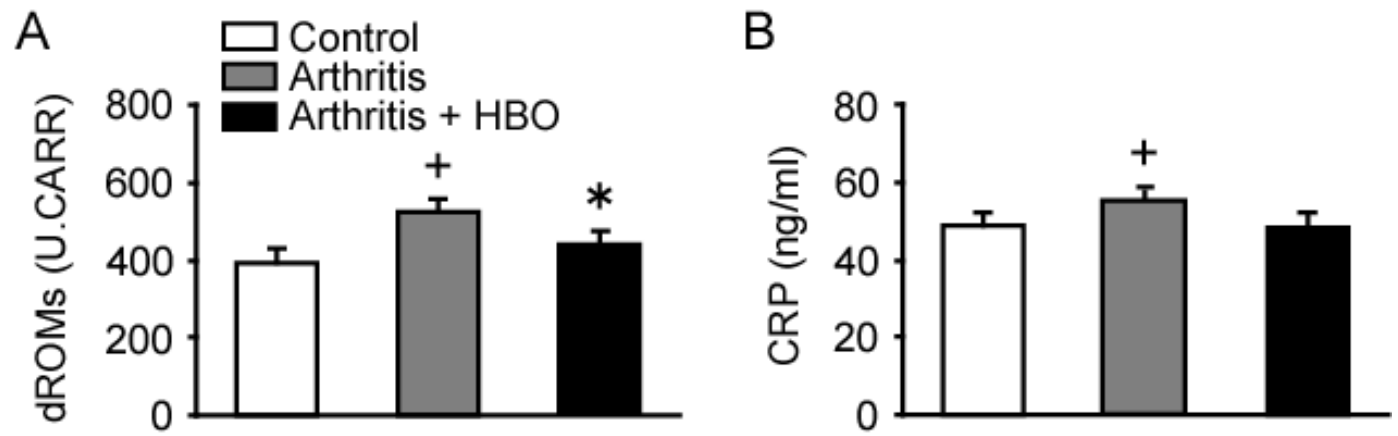

Fig. 3 The serum levels of derivatives of reactive oxygen metabolites $(A)$ and C-reactive proteins $(B)$ in control rats and arthritic rats that were and were not exposed to 
hyperbaric oxygen. Data are presented as mean and standard deviation as determined from 6 animals. dROMs, derivatives of reactive oxygen metabolites;

U.CARR, unit of Carratelli, which is a conventional unit named after an Italian biologist who developed a scale based on the observations from a group of more than 5,000 non-smoking healthy subjects with age ranging from 14 to 80 years ( 1 U.CARR $=0.08$ $\mathrm{mg}$ of hydroperoxide/100 ml hydrogen peroxide); CRP, C-reactive protein; HBO, hyperbaric oxygen exposure. $\quad{ }^{*} P<0.05$ compared to control rats; ${ }^{+} P<0.05$ compared to control and arthritis $+\mathrm{HBO}$ rats.

\section{Serum levels of TNF- $\alpha$, IL-6, and CRP}

Neither TNF- $\alpha$ nor IL- 6 was detected in the rats, irrespective of whether they were arthritic and were exposed to hyperbaric oxygen. The serum level of CRP in the arthritis group was higher than those in the control and arthritis + HBO groups (Figure 3B). No difference in the serum level of CRP was observed between the control and arthritis + HBO groups.

\section{Discussion}

\section{Type II collagen-induced arthritis}

Mice and rats with type II collagen-induced arthritis are widely used as experimental animal models of inflammatory polyarthritis with clinical and pathological features similar to those of rheumatism $[15,16]$. The DA rats are susceptible to induction of adjuvant arthritis. Arthritis is observed in $74 \%$ and $100 \%$ DA rats at 2 weeks and 3 weeks after immunization, respectively (SLC Inc.; Tokyo, Japan). The body weights of DA rats are observed to gradually decrease following immunization; the body weights are the lowest at 3 weeks 
after immunization and subsequently recover to the control level (SLC Inc.). In this study, we examined certain parameters of DA rats at 3 weeks after immunization because all immunized rats demonstrated high severity of arthritis, including the signs of knuckle swelling and/or arch edema (Fig. 2, B1-6). These arthritic rats may undergo further changes leading to cartilage and bone destruction when studied for a prolonged period; similar results were observed in a previous study using mice with type II collagen-induced arthritis [17].

\section{Serum level of dROMs in arthritic rats}

Oxidative stress is the condition in which the production of oxidants exceeds the capacity to neutralize them. Several factors could be involved in the generation of oxidative stress in inflammatory joints $[18,19] . \quad$ ROS are produced during many metabolic processes, including mitochondrial respiration and enzyme activities. ROS concentrations are regulated by maintaining the balance between their production and elimination by antioxidants. An appropriate balance is crucial for normal cell and tissue function. In contrast, the excessive production of ROS damages cellular components, including nucleic acids, lipids, proteins, membranes, and constituents of the extracellular matrix such as proteoglycans and collagens [2]. Previous studies [20, 21] have reported that excessive production of ROS causes an accelerated damage to joint cartilage and osteoclast activation. We observed that the serum levels of dROMs, which serve as a marker of oxidative stress, were higher in arthritic rats than in control ones (Fig. 3A); this finding suggests that arthritis induces an excessive production of ROS. 


\section{Serum levels of TNF- $\alpha$, IL-6, and CRP in arthritic rats}

Several proinflammatory cytokines (TNF- $\alpha$, IL-1, IL-6, and IL-8) are produced by synovial cells and recruit neutrophils to the synovial fluids in arthritic rats. However, we detected neither TNF- $\alpha$ nor IL-6 in the DA rats with arthritis. Previous studies $[22,23]$ have reported increased levels of TNF- $\alpha$ and IL-1 $\beta$ in Wistar or Lewis rats with type II collagen-induced arthritis. We were unable to elucidate the reason underlying the inability to detect cytokines in arthritic DA rats.

We observed increased serum levels of CRP in arthritic rats (Fig. 3B). Previous studies $[24,25]$ have reported that the serum level of CRP correlated with that of proinflammatory cytokines such as IL-6. Increased serum levels of CRP are observed in inflammatory diseases [26]. Therefore, the increased serum levels of CRP reflect the arthritis severity, including signs of knuckle swelling and/or arch edema (Fig. 2, B1-6).

\section{Major drugs for arthritis}

It is widely accepted that reduction of oxidative stress by superoxide dismutase (SOD) affects the course of inflammation. Several studies [27-30] have focused on synthetic low-molecular weight compounds that mimic the effects of SOD. Among the various families of SOD mimetics, the most promising are nitroxides (tempol) and Mn (II) pentaazamacrocyclic ligand (M40403). Tempol diminishes hydroxyl radical production and decreases the cytotoxic effects of hydrogen peroxide and peroxynitrite [27]. Furthermore, it decreases inflammation and tissue damage in rats with type II collagen-induced arthritis. M40403 decreases the release of proinflammatory cytokines such as 
TNF- $\alpha$, probably by inhibiting the expression of the transcription factor, nuclear factor (NF)-KB [28]. The beneficial effects of M40403 have been reported in rats with collagen-induced arthritis [29, 30].

Treatment with 3,7,11,15-tetramethyl-2-hexadecene-1-ol (phytol) increases oxidative burst in vivo, decreases the autoimmune response, and ameliorates both the acute and chronic phase of arthritis in rats [31]. Furthermore, treatment with alpha-lipoic acid (LA), which is a co-factor for mitochondrial a-keto dehydrogenase complexes and which participates in S-O transfer reactions, suppresses the development of collagen-induced arthritis in mice. A previous study [32] reported that the amelioration of joint diseases by LA treatment was associated with reduction in oxidative stress, as well as with the inhibition of inflammatory cytokine activation and NF-kB DNA binding activity. Furthermore, LA-induced decreased intracellular ROS in lymphocytes obtained from the inguinal lymph nodes of arthritic mice and prevented bone destruction in vivo and osteoclastogenesis in vitro.

\section{Effects of exposure to hyperbaric oxygen}

Hyperbaric oxygen therapy leads to new vasoconstriction and hyperoxygenation, making it an effective treatment option for various clinical disorders [33-35]. This treatment involves administration of $100 \%$ oxygen concentration at a pressure greater than atmospheric pressure at sea level, usually equivalent to 2-3 ATA. However, a standard procedure of hyperbaric oxygen therapy is known to cause excessive production of ROS in several tissues and organs $[33,34]$, suggesting that oxidative stress, which is induced by hyperbaric oxygen therapy, accelerates the tissue damage. 
We determined that a pressure of 1.25 ATA and an oxygen concentration of $36 \%$ are required for obtaining effective responses with regard to oxidative metabolism $[7,8]$. In this study, we exposed the rats to moderate atmospheric pressure and oxygen concentration as compared to those exposed to classical hyperbaric oxygen therapy with $100 \%$ oxygen concentration at 2 ATA; we observed that these conditions of hyperbaric oxygen exposure inhibit the excessive production of ROS. Furthermore, these conditions of hyperbaric oxygen exposure are more cost-effective and safe; i.e. treatment at these conditions is not associated with the risk of accidents such as eardrum split. We observed that an excessive production of ROS in spontaneously hypertensive rats was inhibited by exposure to hyperbaric oxygen with $36 \%$ oxygen concentration at 1.25 ATA [36].

Intraarticular pressure is high in inflammatory joints because of decreased compliance of the joint wall, which is attributable to synovial membrane swelling and capsule fibrosis [37]. Other factors contributing to increased intraarticular pressure include movement and accumulation of synovial fluids in chronically involved joints [10]. Together with the reduced capillary density, this elevated pressure could decrease the rate of capillary blood flow and induce lowering of oxygen tension in synovial fluids and repetitive ischemia-reperfusion injury in the inflammatory joint [11-13]. This suggests that arthritic joints are characterized by hypoxia, which is caused by increased oxygen demand and decreased blood flow induced by increased intraarticular pressure. These observations show that a potential therapeutic approach for treating arthritis would be to enhance the partial pressure of oxygen and increase the levels of dissolved oxygen for the elimination of ROS. An increase 
in atmospheric pressure and oxygen concentration enhances the partial pressure of oxygen; this increases the oxygen tension in the affected tissues and the concentration of dissolved oxygen in the plasma, thus enhancing the activity of oxidative enzymes in the mitochondria and, consequently, the rate of oxidative metabolism in cells and tissues. Previously, we reported that the beneficial effects of hyperbaric oxygen exposure with $36 \%$ oxygen concentration at 1.25 ATA were associated with an increased oxidative enzyme activity in skeletal muscle fibers and spinal motoneurons in rats $[7,8]$. In addition, the possible beneficial effects of exposure to hyperbaric oxygen have been reported; the increase in blood glucose level, which is related to growth, of type 2 diabetic rats has been reported to be inhibited by exposure to hyperbaric oxygen [38-40]. We observed that the levels of dROMs decreased in arthritic rats following exposure to hyperbaric oxygen (Fig. 3A); this indicated that the conditions of hyperbaric oxygen exposure used in this study are effective for decreasing the activity of ROS, which are overproduced in arthritic rats. However, further studies are warranted to elucidate whether these effects of exposure to hyperbaric oxygen persist over prolonged periods. In addition, clinical trials must be performed to evaluate the efficacy of exposure to hyperbaric oxygen for the treatment of arthritic patients.

The arthritis scores did not differ between the arthritic rats that were and were not exposed to hyperbaric oxygen (Fig. 2). This result indicates that the effects of exposure to hyperbaric oxygen on the dROM and CRP levels (Fig. 3) did not correspond with those on the morphological profiles of individual paws in arthritic rats. Morphological effects of exposure to hyperbaric oxygen on rats may be observed if they were treated for a prolonged period; in this study, we 
sacrificed the rats only 3 weeks after immunization. Further studies are required to clarify time-dependent effects of exposure to hyperbaric oxygen on the morphological profiles of paws in arthritic rats.

\section{Conclusion}

A minor but significant decrease was induced in the serum levels of dROMs and CRP in arthritic rats after exposure to hyperbaric oxygen. We conclude that the conditions of hyperbaric oxygen exposure used in this study are effective to decrease the activity of ROS, which are overproduced at an early stage of arthritis.

\section{References}

1. Miletić T, Kovačević-Jovanović V, Vujić V et al (2007) Reactive oxygen species (ROS), but not nitric oxide (NO), contribute to strain differences in the susceptibility to experimental arthritis in rats. Immunobiology 212 : $95-105$

2. Henrotin Y, Kurz B, Aigner T (2005) Oxygen and reactive oxygen species in cartilage degradation: friends or foes? Osteoarthritis Cartilage 13: 643-654

3. Henrotin YE, Bruckner P, Pujol JP (2003) The role of reactive oxygen species in homeostasis and degradation of cartilage. Osteoarthritis Cartilage 11: $747-755$

4. Terkeltaub R, Zachariae C, Santoro D et al (1991) Monocyte-derived neutrophil chemotactic factor/interleukin-8 is a potential mediator of crystal-induced inflammation. Arthritis Rheum 34: 894-903

5. Kamezaki F, Yamashita K, Kubara T et al (2008) Derivatives of reactive 
oxygen metabolites correlates with high-sensitivity C-reactive protein. J Atheroscler Thromb 15: 206-212

6. Hirose H, Kawabe H, Komiya N, Saito I (2009) Relations between serum reactive oxygen metabolites (ROMs) and various inflammatory and metabolic parameters in a Japanese population. J Atheroscler Thromb 16: $77-82$

7. Ishihara A, Kawano F, Okiura T et al (2005) Hyperbaric exposure with high oxygen concentration enhances oxidative capacity of neuromuscular units. Neurosci Res 52: 146-152

8. Matsumoto A, Okiura T, Morimatsu F et al (2007) Effects of hyperbaric exposure with high oxygen concentration on the physical activity of developing rats. Dev Neurosci 29: 452-459

9. Hitchon CA, El-Gabalawy HS (2004) Oxidation in rheumatoid arthritis. Arthritis Res Ther 6: 265-278

10. Taylor PC, Sivakumar B (2005) Hypoxia and angiogenesis in rheumatoid arthritis. Curr Opin Rheumatol 17: 293-298

11. Woodruff T, Blake DR, Freeman J et al (1986) Is chronic synovitis an example of reperfusion injury? Ann Rheum Dis 45: 608-611

12. Cross CE, Halliwell B, Borish ET et al (1987) Oxygen radicals and human disease. Ann Intern Med 107: 526-545

13. Blake DR, Merry P, Unsworth J et al (1989) Hypoxia-reperfusion injury in the inflamed human joint. Lancet 11: 289-293

14. Nagatomo F, Gu N, Fujino H et al (2009) Skeletal muscle characteristics of rats with obesity, diabetes, hypertension, and hyperlipidemia. J Atheroscler Thromb, accepted 
15. Trentham DE, Townes AS, Kang AH (1977) Autoimmunity to type II collagen: an experimental model of arthritis. J Exp Med 146: 857-868

16. Courtenay JS, Dallman MJ, Dayan AD et al (1980) Immunisation against heterologous type II collagen induces arthritis in mice. Nature 283: 666-668

17. Okiura T, Nagatomo F, Gu N et al (2008) Bone density of the femur and fiber cross-sectional area and oxidative enzyme activity of the tibialis anterior muscle in type II collagen-induced arthritic mice. J Physiol Sci 58: 221-227

18. Jikimoto T, Nishikubo Y, Koshiba M et al (2001) Thioredoxin as a biomarker for oxidative stress in patients with rheumatoid arthritis. Mol Immunol 38: 765-772

19. Jaswal S, Mehta HC, Sood AK, Kaur J (2003) Antioxidant status in rheumatoid arthritis and role of antioxidant therapy. Clin Chim Acta 338: $123-129$

20. Goldring SR (2002) Pathogenesis of bone erosions in rheumatoid arthritis. Curr Opin Rheumatol 14: 406-410

21. Miossec $P$ (2004) An update on the cytokine network in rheumatiod arthritis. Curr Opin Rheumatol 16: 218-222

22. Salvemini D, Mazzon E, Dugo L et al (2001) Amelioration of joint disease in rat model of collagen-induced arthritis by M40403, a superoxide dismutase mimetric. Arthritis Rheum 44: 2909-2921

23. Jiménez-Caliani AJ, Jiménez-Jorge S, Molinero P et al (2005) Dual effect of melatonin as proinflammatory and antioxidant in collagen-induced arthritis in rats. J Pineal Res 38: 93-99

24. Houssiau FA, Devogelaer JP, Van Damme J et al (1988) Interleukin-6 in synovial fluid and serum of patients with rheumatoid arthritis and other 
inflammatory arthritides. Arthritis Rheum 31: 784-788

25. Madhok R, Crilly A, Watson J, Capell HA (1993) Serum interleukin 6 levels in rheumatoid arthritis: correlations with clinical and laboratoryt indices of disease activity. Ann Rheum Dis 52: 232-234

26. Yeh ETH (2004) CRP as a mediator of disease. Circulation 109 (suppl II): $11-14$

27. Cuzzocrea S, McDonald MC, Mota-Filipe H et al (2000) Beneficial effects of tempol, a membrane-permeable radical scavenger, in a rodent model of collagen-induced arthritis. Arhtritis Rheum 43: 320-328

28. Dai L, Claxson A, Marklund SL et al (2003) Amelioration of antigen-induced arthritis in rats by transfer of extracellular superoxide dismutase and catalase genes. Gene Ther 10: 550-558

29. Cuzzocrea S, Mazzon E, di Paola R et al (2005) Synergistic interaction between methotrexate and a superoxide dismutase mimetic. Pharmacologic and potential clinical significance. Arthritis Rheum 52: 3755-3760

30. Zhou H, Wong YF, Wang J et al (2008) Sinomenine ameliorates arthritis via MMPs, TIMPs, and cytokines in rats. Biochem Biophys Res Commun 376: $352-357$

31. Hultqvist M, Olofsson P, Gelderman KA et al (2006) A new arthritis therapy with oxidative burst inducers. PLoS Med 3: 1625-1636 (e348)

32. Lee EY, Lee CL, Lee K et al (2007) Alpha-lipoic acid suppresses the development of collagen-induced arthritis and protects against bone destruction in mice. Rheumatol Int 27: 225-233

33. Tibbles PM, Edelsberg JS (1996) Hyperbaric-oxygen therapy. N Eng J Med 334: $1642-1648$ 
34. Leach RM, Rees PJ, Wilmshurst P (1998) ABC of oxygen. Hyperbaric oxygen therapy. BMJ 317: 1140-1143

35. Caplan ES (2000) Hyperbaric oxygen. J Pediatr Infect Dis 19: 151-152

36. Nagatomo F, Fujino H, Takeda I, Ishihara A (2009) Effects of hyperbaric oxygenation on blood pressure levels of spontaneously hypertensive rats. Clin Exp Hypertens, accepted

37. Jayson MI, Dixon AS (1970) Intra-articular pressure in rheumatoid arthritis of the knee. 3. Pressure changes during joint use. Ann Rheum Dis 29: 401-408

38. Yasuda K, Aoki N, Adachi T et al (2006) Hyperbaric exposure with high oxygen concentration inhibits growth-associated increase in the glucose level of diabetic Goto-Kakizaki rats. Diabetes Obes Metab 8: 714-715

39. Matsumoto A, Nagatomo F, Yasuda K et al (2007) Hyperbaric exposure with high oxygen concentration improves altered fiber types in the plantaris muscle of diabetic Goto-Kakizaki rats. J Physiol Sci 57: 133-136

40. Yasuda K, Adachi T, Gu N et al (2007) Effects of hyperbaric exposure with high oxygen concentration on glucose and insulin levels and skeletal muscle-fiber properties in diabetic rats. Muscle Nerve 35: 337-343 